\title{
Neutrophilic Eccrine Hidradenitis
}

National Cancer Institute

\section{Source}

National Cancer Institute. Neutrophilic Eccrine Hidradenitis. NCI Thesaurus. Code C112211.

Painful red or pink edematous nodules most commonly found on the palmar and/or plantar regions. 